\title{
A Video Game Level Analysis Model Proposal
}

\author{
Samuel Almeida ${ }^{1,2}$, Ana Veloso, Óscar Mealha \\ ${ }^{1}$ CETAC.MEDIA, Dept. of Communication and Art \\ University of Aveiro \\ Aveiro, Portugal \\ sja@ua.pt,aiv@ua.pt,oem@ua.pt
}

\author{
Licínio Roque, Arnaldo Moura \\ ${ }^{2}$ CISUC, Dept. of Informatics Engineering \\ University of Coimbra \\ Coimbra, Portugal \\ lir@dei.uc.pt, arnaldo@student.dei.uc.pt
}

\begin{abstract}
This paper presents a preliminary model proposal for the analysis of video game level scenarios consisting of four elements: video game, players, metrics, and gaming experience. The selection of parameters from each of these elements defines a specific video game level analysis context. Initial validation of the model is being carried out with a First-person shooter video game and selected metrics (including eye movement behavior) to understand player behavior (both interactive and visual) within a game level. An additional analysis technique based on visual representation techniques applied in this validation context is also described. After validated, this model may be applied in the analysis of player behavior in game levels, in several different situations predicted in the model itself. Information acquired from the application of this model may posteriorly be used in the design (or redesign) of game levels and scenarios driven for optimal use which should generate a greater gaming experience.
\end{abstract}

Keywords - Video Game Level, Analysis Mode, Gameplay Metrics, Eye Tracking, Level Design, Gaming Experience, Player

\section{INTRODUCTION}

Level design - "the act of creating a piece of a greater whole" [1] - is a crucial part of the game development process as it results in the creation of a stage where the action takes place. When a game development team creates a game (and game levels), the end goal is for players to explore it to its full extent. However, studies [2, 3] have shown that in particular 3D video game levels, this does not always happen. In some cases, up to $25 \%$ of a game level may be overlooked by players. While different playing styles may impact how much of a game a player takes advantage of, and, depending on the game genre and objectives; it is conceivable for players to complete or go through games without having interacted with part of the game elements (scenery, objects or even characters), some of which are crucial to the actual flow of gameplay. Therefore, if game development teams understood player behavior - namely movement patterns, interactions with other non-playable characters and level entities, as well as eye movement behavior - level designers and artists could conceive levels that appeal more to the player and be more exhaustively and adequately explored.

This paper proposes a solution for this gameplay and level exploration and interaction issue, by presenting a preliminary model proposal for video game level analysis consisting of four elements: video game; players, metrics; and gaming experience. The selection of parameters from each of these elements defines a specific analysis context from which data can be extracted regarding player behavior within game levels. The variety of combinations of gameplay and psychophysiological metrics can result in information that may posteriorly help designers, developers and researchers reflect on level design issues; e.g. areas of a level that are explored the most/least, movement patterns for a single player or a team, the actions and events players execute in various game situations, among others.

\section{RELATED WORK}

The development and application of models in game studies has covered multiple areas. Sweetser \& Wyeth [4] proposed a model - 'GameFlow' - for evaluating player enjoyment in games based on eight elements; Fabricatore et al. [5] presented a model for the understanding of playability of video games; Nacke [6] proposed a hierarchical game usability model based on research regarding usability approaches in video games. Despite these examples, research has not focused on models that solve game level questions based on player behavior that can be recorded and analyzed using metrics.

The use of metrics in game analysis is a recent approach. The term 'metric' originates from computer science and refers to a standard unit of measure. Metrics are used in the evaluation and measurement of processes, events and interactions [7]. The use of (gameplay) metrics works by recording User Initiated Events (UIEs) or player behavior, resulting in log files with player related data [8] regarding interaction within or with a virtual environment (VE). Additionally, these events and behaviors may be initiated by agents or other systems present in the VE, not under the influence of the player [7]. Further player behaviors that may be considered as metrics are those of psychophysiological nature. Game metrics may be categorized according to several scopes: (1) temporal resolution [9], (2) spatial resolution [10], and (3) level of abstraction.

Regarding temporal resolution (1), the recording of metrics can be of three types: (i) continuous; (ii) based on a defined frequency (e.g. measurement of a player event every 2 seconds) or (iii) triggered (e.g. record a specific event every time it occurs) [9]. Concerning spatial resolution (2), metrics can be (i) spatial (if they operate in the spatial environment of a game world and have an $x, y, z$ coordinate association) or (ii) non-spatial (if no $x, y, z$ coordinate is associated) [10]. Non-spatial metrics do not contain any spatial information. Lastly, concerning their level of 
abstraction (3), metrics can be (i) low-level (e.g. logging keystrokes) or 'high-level' (player movement throughout a game) [9].

The use of gameplay metrics is a novel approach but is also rapidly growing. Kim et al. [8] used metrics along with survey and video data to understand player behavior and improve game design, describing their use in two case studies; Drachen \& Canossa [11] applied metrics to analyze gameplay during game production, presenting a series of case studies; Tychsen \& Canossa [9] explored the use of gameplay metrics to describe personas of players (patterns of play).

As occurs with gameplay metrics, the use of eye tracking in a video game context for analysis purposes is still in an embryonic stage, with only a handful of occurrences to account for $[2,3,12-16]$. Eye tracking and visual attention patterns in game contexts can help improve the design of game levels, decreasing frustration [12] and increasing the gaming experience. Eye tracking can inform on where players are looking while playing a game and for what period of time. These two elements can be later used by developers and designers so they know "where to place items in a level or how to choose colors and use other visual tools to stimulate attention and eliminate (...) problems" [12].

El-Nasr \& Yan [12] applied eye tracking to explore whether players' visual attention patterns follow the 'bottomup' or 'top-down' visual attention theories; Johansen et al. [14] explored how eye tracking could address several challenges faced by a computer game developer in their pursuit to develop better, more usable, fun and challenging games. Almeida [2] used eye tracking to analyze how players visually interacted with video game scenarios. He developed a method that helped identify game level areas that were and were not visualized by players. In another study, Almeida [3] explored the differences in visual and interaction behavior between hard core and inexperienced players. Regarding gaming experience, Jennet and colleagues [13] were able to use eye tracking to determine that the intensity of eye movements varies according to level of immersion in which a player is in.

\section{Study Aims \& RELEVANCE}

The study described in this paper has the following main goal which is twofold: (i) present a preliminary video game level analysis model consisting in four elements: video game, players, metrics, and gaming experience; and (ii) present the setup of a first validation of the proposed model.

A first validation of this model is being carried out according to a defined analysis context in which parameters from several of the model elements have been identified. Specifically, for the metrics element, data such as player events, position and eye movement behavior is considered. These metrics were selected because they can help represent player interaction within a game level. The way in which players move within a game level; the way players interact with other players and non-playable characters and entities, may provide information about the level design. Eye movement behavior provides insight regarding what aspects of a game level attracted players' visual attention and can posteriorly help designers understand if gameplay elements were visualized.

The relevance of this study is connected to some evidence presented in the introductory section. Studies [2, 3] have demonstrated that in some contexts, significant areas of a 3D game level can go unnoticed or without exploration by players, independently of their experience with videogames. Fig. 1 represents a heat map illustrating hardcore player's visual and interaction behavior [2], also related to level exploration.

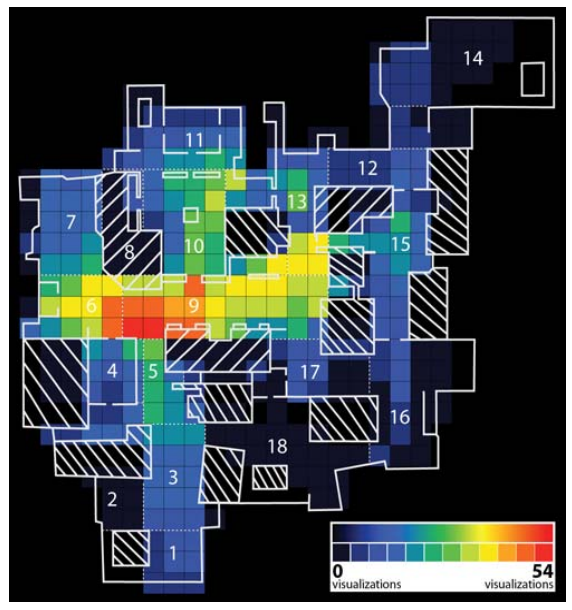

Figure 1. Heat map representation of hardcore players' visual and interaction behavior

This heat map representation indicates that for a specific level, a significant percentage of the map was poorly explored (areas represented in 'colder' colors). Areas with 'warm' colors are the most explored. Because players are a game's end target, understanding player input - in the form of gameplay data as well as eye movement - is essential. Consequently, video game levels can be improved, even if slightly, if player tendencies, opinions and direct input are taken into consideration.

The multiplicity of defined metrics may offer resourceful information regarding player behavior; scenic and level elements that might have played part in a determined behavior, event or action; it can inform on what areas of the map are explored the most/least and how players populate the map; how and with what map/level entities and nonplayable characters players interact with; if essential game elements or artifacts are being used by players, some of which are essential to the flow of gameplay. If the model is applied during game development, game designers can rectify design issues before the game is finished and released. If applied in a later stage of the development process, the data can be used as a reference in the development of future games of the genre.

\section{VIDEO GAME LEVEL ANALYSIS MODEL}

The Video Game Level Analysis Model (VGLAM) described in this paper consists in four elements; these are: (1) Video Game; (2) Players; (3) Metrics; and (4) Gaming 
Experience. The definition and selection of parameters within each of the elements defines a specific analysis context that can help in the analysis of desired aspects of video game levels. Fig. 2 represents the specified VGLAM.

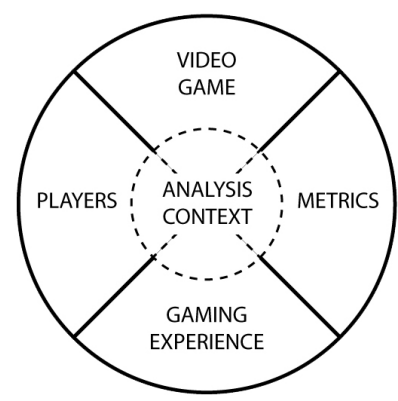

Figure 2. Representation of the Video Game Level Analysis Model

The first of the elements, (1) 'Video Game', describes the genre of games that this model can analyze. There are many types of game genres, divided according to several taxonomies [17, 18]. However, this model may primarily support a spectrum of genres such as: First-Person Shooters (FPS), Third-Person Shooters (TPS), Role-playing games (RPG), Real-time Strategy (RTS) and some simulation games. In common, these genres share the fact that their gameplay includes at least one playable character, takes place in one or more game levels (larger or smaller in size) and promotes interaction with one or more Non-playable characters (NPC) and other entities. Ideally, for each genre, respective $3 \mathrm{D}$ game levels can be projected as $2 \mathrm{D}$ maps where the gameplay takes place. This 'map' will later serve as a georeference layer on which data will be analyzed.

The second element, (2) Players, describes an element that is crucial to all video games - their end target. Video games are designed and developed for players, and to entertain players. Players and the act of play and player behavior (interaction and visual) in games are dependent of many variables: motivations, skills, past experience and expected experiences, for example. Crawford [17] speaks extensively on the motivation to play games. This author highlights the motivation to learn as a main factor for playing, even if a player is not conscious of that motive. For other players, motivation might reside in other factors such as the need to explore, to prove oneself or the need for acknowledgement. Furthermore, player gender and age may also play a role in motivation. Skills are also a relevant factor in the player element, and are closely related to another of the model elements (Gaming Experience) [19]. A player's skills in a specific game can influence the outcome and the success in a game. Skills can also influence a player's motivation to continue playing a game. Lastly, players' past and expected experiences also play a role in a player's performance in a game, and can also influence motivation.

The third element, (3) 'Metrics', describes gameplay and other behavior metrics that are used in a defined analysis context. As explored in the 'Related Work' section, metrics can be categorized in multiple ways. Furthermore, these may be analyzed independently or in relation to others. The complexity of each game genre will define the number and type of metrics that can be analyzed. For example, a FPS game may offer gameplay metrics that cannot be found in a Sports Simulation game and vice-versa. Despite these possible variances, there are metrics that exist independently of the game genre in analysis and span across all defined game genres, for example: time, player-character actions/events, $x, y, z$ coordinates for a player-character, nonplayable characters and level entities. Table 1 presents a list of some game metrics that may be used in game level analysis for two game genres: FPS and Sports (Racing) Simulation. Table 1 also specifies how several game metrics may have different applications and meaning in two different game genres.

TABLE 1. Examples of game metrics for two types of games

\begin{tabular}{|c|c|c|}
\hline Metric & FPS & $\begin{array}{c}\text { Sports (Racing) } \\
\text { Simulation }\end{array}$ \\
\hline Time & $\begin{array}{c}\text { Time to } \\
\text { complete a } \\
\text { mission }\end{array}$ & $\begin{array}{c}\text { Time spent on a } \\
\text { lap }\end{array}$ \\
\hline Energy & $\begin{array}{c}\text { Remaining } \\
\text { running boost }\end{array}$ & Remaining fuel \\
\hline $\begin{array}{c}\text { Player } \\
\text { Action }\end{array}$ & $\begin{array}{c}\text { E.g. jumping, } \\
\text { running }\end{array}$ & Accelerate/Break \\
\hline Team & $\begin{array}{c}\text { Blue Team/Red } \\
\text { Team }\end{array}$ & Ferrari/Renault \\
\hline $\begin{array}{c}x, y, z \\
\text { coordinates }\end{array}$ & $\begin{array}{c}\text { Current position } \\
\text { of player- } \\
\text { character in } \\
\text { level }\end{array}$ & $\begin{array}{c}\text { Current position } \\
\text { of car on track }\end{array}$ \\
\hline
\end{tabular}

Other data considered in this model is that directly related to the player and of psychophysiological nature: eye tracking (eye movement and behavior); electromyography (EMG); cardiovascular measures and others. In the case of eye tracking, quantitative data regarding where and how a player was looking at a certain object can provide insight about, for example, what level entities are visualized with greater or less intensity. Lastly, a sub-element of the 'Metrics' element is the 'In-Game Environmental' metrics. These metrics deal with specific level parameters that can be altered and that can produce an effect on other gameplay metrics. Examples of these 'in-game environment' metrics are the size of the level (map), quantity of resources/entities and climate conditions. Furthermore, 'In-Game Environmental' metrics can have an effect on another of the elements described in the model: gaming experience.

The fourth element of the model, (4) 'Gaming Experience', deals with a selection of traits that may potentially be analyzed with the model; e.g.: immersion and flow. Immersion and flow are two of the most common gaming experiences and have been studied extensively [13, $16,19-22]$. The quality of the gaming experience can be a direct result of the quality of a level and is therefore a relevant element of the model. Game experiences as a whole 
may not fully be understood using the model, but several gameplay (e.g. number of deaths, player movements) and psychophysiological metrics (e.g. eye tracking) may provide an understanding of the quality of a level. For example, in a shooting game, a low number of deaths may indicate that the game is too easy, suggesting a more boring game and therefore, hindering a positive flow state. Additionally, as referred, the variation of in-game environmental metrics can also influence the quality of the gaming experience. To exemplify, in a racing game, an insufficient time to complete a race may cause a player to become anxious, also hindering a positive flow experience.

The four elements of the proposed model are all connected and interdependent. A video game is a central element of the model and can only exist if there are players to play the game. Different player profiles, based on motivations, skills and experiences, will influence players' behavior within the game. The game and game genre can also influence player behavior. The qualities of a game and a player profile can define the quality of the gaming experience. Metrics can be associated to games and players: gameplay metrics in the case of games and psychophysiological metrics in the case of players. Lastly, metrics can provide valuable information regarding player behavior with and within a game. These diverse relations among elements establish the interdependency of the model. The selection of parameters from these elements defines a selected 'analysis context' from which information can be collected, correlated and analyzed. This correlation and analysis can help establish connections between each of the parameters defined in the model.

In some contexts, the analysis of quantifiable data is not linear. Therefore, further techniques and/or methods can be applied that assist in establishing a connection between parameters (e.g. gameplay metrics). For example, a linear correlation of eye tracking data and player movement is not direct. As a result, additional techniques may be used to combine these two metrics and assist in understanding the acquired information; e.g. the relation between where a player was moving and where he was looking. Visual-based techniques are one option to assist in the analysis of data from the metrics defined in the analysis context. Depending on the specificities of each context, multiple visual analysis techniques may be used. For example, heat maps - which use color to represent levels of intensity - can be used to analyze multiple metrics (e.g.: areas where player movement was most/least intense; areas where player interactions were most frequent). Clustering can also visually assist in the analysis of metrics within defined areas (clusters) of a game level (e.g. providing information regarding number of deaths in various areas of a map, in a FPS). Furthermore, common visual representations such as bar graphs and pie charts can assist in visually interpreting two or more metrics (e.g. time and number of interactions with other non-playable characters).

In a game/level design process, this model can be used in the analysis of player behavior in game levels. The definition of parameters from each of these elements - a game and genre, a player(s) profile and metrics - provides an analysis context from which information can be extracted. This information can be later analyzed and interpreted with the help of one or more analysis techniques. In terms of applicability during the game development process, it is expected that the model can be applied during game development or used in post-launch analysis, with different impacts. Globally, the model can help understand and analyze how players interact within their game level; it can help understand if player interaction patterns are those that are expected; if players are moving through the game and interacting with crucial gameplay elements, which can ultimately define the quality of their gaming experience.

\section{Model VALIDATION}

A first validation of the Video Game Level Analysis Model is being carried out in order to confirm the relevance of each of the constituent elements.

An analysis context has been defined based on the selection of parameters from several of the elements that make up the model: a video game was developed (described in section $5 \mathrm{~A}$ ) for the purpose of this validation and game metrics were defined according to possibilities of the game. However, gaming experiences have not been initially considered. Additionally, in this first validation, one of the metrics selected was of psychophysiological nature: eye movement (visual behavior). The selection of the eye movement metric resulted in the need to apply an additional technique that was used to collect, extract and subsequently, interpret data. The analysis of eye movement in dynamic media (i.e. video games) cannot be carried out using visualization techniques (e.g. gaze plots, bee swarms, clusters) frequently used with static media (e.g. pictures) [23]. As a result, a specific technique was projected in order to carry out the intended collection of data for posterior analysis. Fig. 3 is a schematization of the technique used during the validation process, consisting in 4 phases: Data Tracking; Log Files; Computing; Data Visualization System.

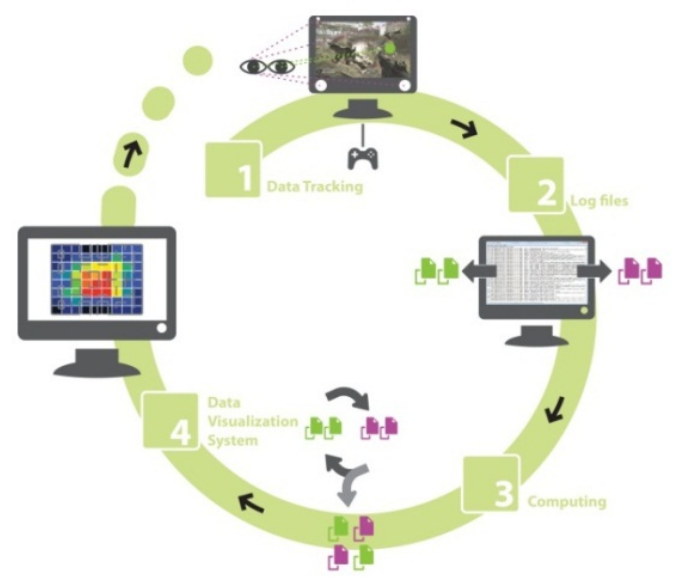

Figure 3. Representation of the technique used in the validation process

The first phase (1), 'Data Tracking', is related to the capturing of player eye movements using an eye tracker while playing the video game. The second phase (2), 'Log 
Files', refers to the extraction of log file data from the eye tracker and video game. Phase three (3) and four (4), 'Computing' and 'Data Visualization System', deal with the integration and correlation of the selected metrics: eye tracking and gameplay metrics. These are then processed with an analysis tool (described in 5B) developed specifically to interpret these combined data. The resulting data may later be analyzed through the use of several information visualization paradigms, such as a heat map (as seen in Fig. 1).

\section{A. Video Game study object}

Regarding the video game developed and used in this first validation; the platform selected for development was 'Unity' (http://unity3d.com/). The game, titled 'ReCycle', has an intentionally simple and flexible design which allows for a multiple number of combinations of game parameters, ultimately leading to various types of gameplay dynamics. Fig. 4 represents a screenshot from 'ReCycle'.

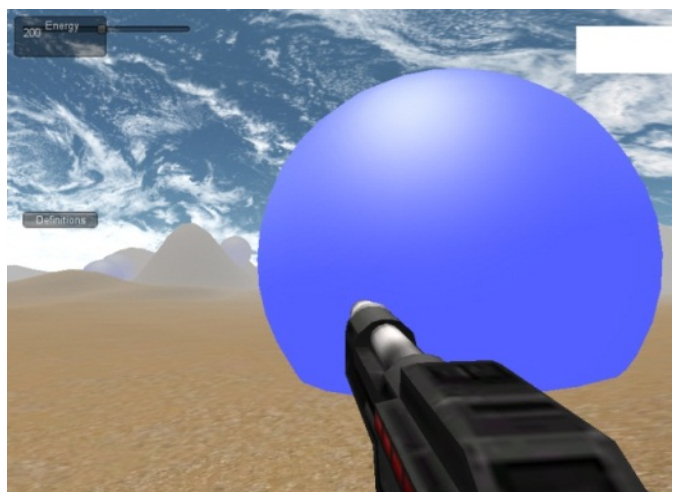

Figure 4. Screenshot from the game 'ReCycle'

The objective of the game is for players to survive in a desert where water is the only source of energy. The game also consists of a day and night cycle that results in energy loss after each cycle. As a result of this, players must look for water (found in the blue spheres in Fig. 4), scattered around the desert, to stay alive. Each player, carrying a weapon, must survive in the desert, fighting off opponents and, in some game variants, may form alliances. These alliances bring both benefits as well as responsibilities. Each gameplay session lasts until there is a single survivor in the desert.

The game logs two types of data: continuous and eventtriggered. Continuous data - written by the server - is logged into a file and contains player positions and status. Every 0.2 seconds the server writes for each player their ID, username, current position, rotation, alliance, energy and the time stamp of this information. A second log file is created with playertriggered events: jumping; shooting, harvesting water, running or alliance proposal.

\section{B. Analysis Tool Development}

An analysis tool was developed and applied in this first validation process which responds to the needs of the predefined analysis context. Although this analysis tool has been developed for a particular analysis context, it intends to have sufficient versatility to be applied in other analysis contexts with different parameters in each of the elements of the model.

The tool initially requires an upload of a 2D map of the game level being analyzed. This map serves as a georeference layer on which additional layers of player activity is represented.

Once the game map is imported, the tool is launched and an initial layer of information is presented. This layer of information consists in the representation of movements by players used in the study. In addition, the analysis tool consists of other functions: (i) Timeline control: controls the evolution of a player's movements and events within the course of a player's gameplay time; (ii) Player selection filters: filters allow the selection of what player's movements and actions to visualize and control; (iii) Player events representation: a user may control what player-triggered events (jumping, shooting, harvesting water, running or alliance proposals) to see independently or simultaneously; (iv) Player health evolution: the evolution of one or more player's health may be visualized and analyzed according to their movements and other player events that influence the increase/decrease of health; (v) Information representation: the combination of log file data regarding gameplay metrics and eye movement data can be represented and analyzed through a combination of different information visualization paradigms. For example, heat maps may be used to represent areas of the map that were explored and populated the most, thereby also revealing eventual problematic areas of the game level. This last function is also connected to the last element of the model, regarding visual-based analysis.

\section{CONCLUSIONS AND FUTURE WORK}

The objective of this paper is to present a preliminary video game level analysis model currently in validation. The model consists of a four elements: Video Game; Players; Metrics; and Gaming Experience. The selection of parameters from each of these connected and interdependent elements defines a specific analysis context.

Currently, a first validation is being carried out using a First-Person shooter game. Several gameplay metrics as well as eye tracking behavior were defined in order to understand player interaction and visual behavior. The definition of these parameters demanded the development of additional techniques that are used in the correlation of these sets of metrics. Nonetheless, further validation of the model is necessary and is being carried out for this analysis context.

Initial results have been applied in the rectification of the model itself. The current model proposal based on four elements has been iteratively refined to its current state, based on the removal of elements and addition of others, namely gaming experience. Further work will result in a more concise Video Game Level Analysis Model.

The validation of the model may ultimately help understand player behavior - interactive and visual - in game levels based on defined gameplay and other metrics. This and other information can help developers make more conscious choices during the game development process or 
in future versions of a game. Additionally, with this knowledge, levels can be conceived and designed for optimal usage, therefore creating a more intense and gratifying gaming experience - the ultimate goal of any video game.

\section{ACKNOWLEDGMENT}

Special thanks to Labs.SAPO of the University of Aveiro for providing the eye tracker used in our studies; and to our colleague Celso Soares. Finally, our acknowledgments to the Portuguese institution 'Fundação para a Ciência e Tecnologia' (FCT) for funding this project with the $\mathrm{PhD}$ grant no. SFRH/BD/66527/2009.

\section{REFERENCES}

[1] B. Bates, Game Design, Second ed.: Thomson Course Technology, 2004.

[2] S. Almeida, "Augmenting Video Game Development with Eye Movement Analysis," M.Sc Thesis, Departamento de Comunicação e Arte, Universidade de Aveiro, Aveiro, 2009.

[3] S. Almeida, Ó. Mealha, and A. Veloso, "Interaction Behavior of Hardcore and Inexperienced Players: "Call of Duty: Modern Warfare" Context " in Proceedings of SBGames 2010 - IX Brazilian Symposium on Computer Games and Digital Entertainment, Florianopolis, SC, Brazil, 2010.

[4] P. Sweetser and P. Wyeth, "GameFlow: a model for evaluating player enjoyment in games," Comput. Entertain., vol. 3, pp. 1-24, 2005.

[5] C. Fabricatore, M. Nussbaum, and R. Rosas, "Playability in action videogames: a qualitative design model," Hum.Comput. Interact., vol. 17, pp. 311-368, 2002.

[6] L. Nacke, "From playability to a hierarchical game usability model," presented at the Proceedings of the 2009 Conference on Future Play on @ GDC Canada, Vancouver, British Columbia, Canada, 2009.

[7] A. Drachen and A. Canossa, "Analyzing user behavior via gameplay metrics," presented at the Proceedings of the 2009 Conference on Future Play on @ GDC Canada, Vancouver, British Columbia, Canada, 2009.

[8] J. H. Kim, D. V. Gunn, E. Schuh, B. Phillips, R. J. Pagulayan, and D. Wixon, "Tracking real-time user experience (TRUE): a comprehensive instrumentation solution for complex systems," presented at the Proceeding of the twenty-sixth annual SIGCHI conference on Human factors in computing systems, Florence, Italy, 2008.

[9] A. Tychsen and A. Canossa, "Defining personas in games using metrics," presented at the Proceedings of the 2008 Conference on Future Play: Research, Play, Share, Toronto, Ontario, Canada, 2008.

[10] A. Drachen and A. Canossa, "Analyzing spatial user behavior in computer games using geographic information systems," presented at the Proceedings of the 13th International
MindTrek Conference: Everyday Life in the Ubiquitous Era, Tampere, Finland, 2009.

[11] A. Drachen and A. Canossa, "Towards gameplay analysis via gameplay metrics," presented at the Proceedings of the 13th International MindTrek Conference: Everyday Life in the Ubiquitous Era, Tampere, Finland, 2009.

[12] M. S. El-Nasr and S. Yan, "Visual attention in 3D video games," presented at the Proceedings of the 2006 ACM SIGCHI international conference on Advances in computer entertainment technology, Hollywood, California, 2006.

[13] C. Jennett, A. L. Cox, P. Cairns, S. Dhoparee, A. Epps, T. Tijs, and A. Walton, "Measuring and defining the experience of immersion in games," Int. J. Hum.-Comput. Stud., vol. 66, pp. 641-661, 2008.

[14] S. A. Johansen, M. Noergaard, and J. Rau, "Can Eye Tracking Boost Usability Evaluation of Computer Games?," presented at the CHI 2008: Evaluating User Experiences in Games, 2008.

[15] T. Lin and A. Imamiya, "Evaluating usability based on multimodal information: an empirical study," presented at the Proceedings of the 8th international conference on Multimodal interfaces, Banff, Alberta, Canada, 2006.

[16] L. Nacke and C. A. Lindley, "Flow and immersion in firstperson shooters: measuring the player's gameplay experience," presented at the Proceedings of the 2008 Conference on Future Play: Research, Play, Share, Toronto, Ontario, Canada, 2008.

[17] C. Crawford. (1984). The Art of Computer Game Design. Available: Digital Copy available at: http://library.vancouver.wsu.edu/sites/library.vancouver.wsu.e du/files/ACGD.pdf

[18] A. Rollings and E. Adams, Andrew Rollings and Ernest Adams on Game Design: New Riders Games, 2003.

[19] M. Csíkszentmihályi. (1990). Flow: The Psychology of Optimal Experience.

[20] J. Chen, "Flow in games (and everything else)," Commun. $A C M$, vol. 50, pp. 31-34, 2007.

[21] J. Takatalo, J. Häkkinen, J. Kaistinen, and G. Nyman, "Presence, Involvement, and Flow in Digital Games," in Evaluating User Experience in Games: Concepts and Methods (Human-Computer Interaction Series), $\mathrm{R}$. Bernhaupt, Ed., First ed: Springer, 2010, p. 288.

[22] E. Brown and P. Cairns, "A grounded investigation of game immersion," presented at the CHI '04 extended abstracts on Human factors in computing systems, Vienna, Austria, 2004.

[23] Ó. Mealha, A. Veloso, S. Almeida, R. Rodrigues, L. Roque, R. Marques, and C. Manteigueiro, "Eye Tracking Data Representation and Visualization: on Information and Communication studies at CETAC.MEDIA," Journal of Eye Tracking, Visual Congnition And Emotion, 2012. 